\title{
Detaching RAD from DSED: the rationale and research requirements
}

\author{
Attachment disorders were defined in the clinical literature $>40$ years ago, but their \\ systematic analysis has only occurred relatively recently. In 2015, Charles Zeanah and \\ Mary Margaret Gleason compiled an Annual Research Review into attachment disorders \\ in early childhood for the Journal of Child Psychology and Psychiatry. Here, the \\ researchers outline the key findings from their review and provide an update as to how the \\ field has progressed over the past years.
}

Attachment disorders characterize severe and impairing disturbances in a young child's relationships with caregivers, and were formally defined in the Diagnostic and Statistical Manual of Mental Disorders-III (DSM-III) in 1980. Since then, their criteria have been revised up until the most recent DSM-5 publication in 2013. DSM-5 describes attachment disorders as two distinct, but related disorders: Reactive Attachment Disorder (RAD) and Disinhibited Social Engagement Disorder (DSED). "This edition of DSM-5 marked an important change as to how attachment disorders are conceptualized", explains Zeanah. "As such, our review aimed to address the evidence supporting the DSM-5 structure reflecting that RAD and DSED are indeed distinct disorders, despite sharing a common aetiology of inadequate care-giving".

The researchers found considerable, consistent evidence to support the distinction between RAD and DSED: although both disorders describe aberrant social behaviours in children exposed to inadequate care-giving (i.e., severe social deprivation and other situations in which a child has limited opportunities to form selective attachments), they differ in most other features. The core features of RAD in children include the absence of focused attachment towards a caregiver, failure to seek and respond to comfort when distressed, reduced social and emotional reciprocity and disturbances in emotional regulation (such as unexplained fearfulness or irritability). Conversely, the core features of DSED in children consist of an inappropriate approach to unfamiliar adults and lack of wariness of strangers, a willingness to wander off with strangers, and a lack of appropriate social and physical boundaries (e.g. actively seeking close physical contact). In addition to their very different clinical presentation, their clinical correlates, course, relation to classifications of attachment, and responsiveness to adequate care-giving environments are quite different.

Despite the positive advance in recognizing these attachment disorders as two different entities, Zeanah and Gleason highlight that many questions remain unanswered. "We still know little about the vulnerability factors that may predispose children to RAD or DSED in conditions of deprivation", explains Zeanah. "Severe neglect is necessary, but not sufficient to cause either disorder; why some children become emotionally withdrawn and inhibited whereas others become socially disengaged is unknown".

Some explanation to this question has started to evolve, based on prenatal and very early infant-mother interaction studies. In their 2015 review, Zeanah and Gleason detailed a study by Oliveira et al. of institutionalized toddlers in Portugal that found that experiences prior to institutionalization predicted indiscriminate behaviour - the hallmark of DSED. Such experiences include maternal prenatal risk, limited prenatal care and preterm birth, and their association with DSED seems to be mediated by neglect. Since the time of the review the same group have studied children in Portugal with RAD and have found that experiences prior to institutionalization also predict RAD, to a greater extent than the quality of the current caregiver-child relationship. "Their data show that having a less selective caregiver- 
child relationship, being institutionalized previously, being exposed to early parental abandonment, and lacking regular contact with the original family at the family home while institutionalized, increases the risk of RAD", says Zeanah. "These findings all underscore the especially toxic effects of institutional rearing in infancy and early childhood". Yet as discussed further in their review, although the majority of maltreated children and children raised in institutions have insecure or disorganized attachments to their caregivers, the majority do not develop attachment disorders. Most adoption studies have agreed, however, that the length of time spent in an institution is associated with risk of DSED.

Zeanah and Gleason predominantly focused their review on RAD and DSED in early childhood but recommended follow-ups in later childhood, adolescence and adulthood that include examining peer relationships and interpersonal competence. They questioned whether social impairments persist long after signs of the attachment disorder appear and if and how the symptoms of affected children might change over the course of childhood and into adolescence. Since the publication of their review, the researchers have used data from the Bucharest Early Intervention Project to shed some light on these issues and examine the course of RAD and DSED signs from early childhood to early adolescence.

"Thus far, we have found that although foster care is associated with steep reductions in the signs of RAD across development, later age of placement into families and greater time spent in institutional care are both associated with prolonged and elevated signs of RAD", describes Zeanah. "Similarly, we have found that children who remain in institutional settings for long periods of time, are placed into families at older ages, and thus experience more family placement disruptions have persistently modest-to-elevated signs of DSED over 10 years from early childhood to early adolescence".

The researchers have also explored social functioning in young adolescents with RAD and DSED. They have found that elevated signs of RAD and DSED are associated with poorer general social functioning as well as poorer social competence, and signs of DSED only, are associated with greater peer conflict and higher scores on caregiver-reported victimization. Their data also suggest that signs of RAD and DSED predict poorer social functioning above and beyond time spent in institutional care and placement disruptions.

Since their review, Zeanah and Gleason have identified several additional studies that have started to disentangle RAD from DSED and started to answer some of the aforementioned unaddressed issues. One such study by Mayes et al. published in Clinical Child Psychology and Psychiatry found no overlap in signs of autism in children with RAD or DSED, and concluded that children can meet criteria for both autism and RAD or DSED. These findings refute the DSM-5 requirement that autism is an exclusionary diagnosis and instead support that there is no reason why a child cannot have both disorders.

Zeanah and Gleason also note an important study by Lawler et al. published in the Journal of Child Psychology and Psychiatry, who observationally assessed signs of DSED four times over the first two years after adoption of infants out of institutions. When these children were five years old, their caregivers were interviewed to determine whether they met the criteria for DSED. "Here, the data showed that post-institutionalized children displayed higher initial levels of disinhibited social engagement behaviours and a steeper rate of increase of such behaviours compared to non-adopted children", explains Zeanah. "This study thus categorizes indiscriminate behaviours into physical and nonphysical (i.e., social) 
manifestations and demonstrates that behavioural trajectories rather than initial presentation of indiscriminate behaviour is most predictive of a categorical diagnosis in young children".

Now, more work remains to identify suitable and effective interventions (beyond high-quality care-giving) that are tailored to each, individual disorder. Thus far, intervention studies for RAD and DSED have been limited, with only one case report of adaptation of parent-child interaction therapy to a preschool child with persistent DSED that was effective in resolving the disorder. No systematic interventions have yet been evaluated. As Zeanah and Gleason discuss, the majority of children with RAD seem to respond to enhanced care-giving, but this approach is only effective in a proportion of those with DSED. The reasons why those with DSED are less responsive to enhanced care-giving are unclear, and unfortunately, alternative interventions to facilitate recovery from DSED have not yet been established.

"Over the next decade, we hope that a better understanding of the neural substrates of RAD and DSED may help delineate the mechanisms linking inadequate care to these disorders and the mechanisms involved in recovery", says Zeanah. "We expect that interventions for DSED will have been tested, as to date, only enhanced care-giving has been examined and although effective for many children, it is not effective for all".

Zeanah and Gleason conclude that although they did not find the results of their 2015 review surprising, it did address some previous controversies, such as how much DSED reflects disturbed attachment as opposed to disturbed social behaviour. It remains unresolved however, whether "approach to strangers" observed in DSED is motivated by attachment or by not being inhibited by strangers.

\section{Original Article}

Zeanah, C. H. \& Gleason, M. M. (2015), Annual Research Review: Attachment disorders in early childhood-clinical presentation, causes, correlates, and treatment. J Child Psychol Psychiatr, 56: 207-222. doi:10.1111/jcpp.12347

\section{Further Reading}

Mayes, S.D., Calhoun, S.L., Waschbusch, D.A. \& Baweja, R. (2016), Autism and reactive attachment/disinhibited social engagement disorders: Co-occurrence and differentiation. Clinical Child Psychology and Psychiatry, 22, 620-631. doi: 10.1177/1359104516678039.

Lawler, J.M., Koss, K.J., Doyle, C.M. \& Gunnar, M.R. (2016).. J Child Psychol Psychiatr, 57:1126-1134. doi: 10.1111/jcpp.12606.

Corval, R., Belsky, J. Baptista, J., Oliveira, P., Mesquita, A. \& Soares, I. (2017), Inhibited attachment disordered behavior in institutionalized preschool children: Links with early and current relational experiences. Attachment and Human Behavior, 19:598-612. doi:10.1080/14616734.2017.1342172. 